\title{
openheart Echocardiography in suspected coronavirus infection: indications, limitations and impact on clinical management
}

\author{
Megan M Sheehan, ${ }^{1}$ Yoshihito Saijo (D) ,2 Zoran B Popovic, ${ }^{3}$ Michael D Faulx (D ${ }^{3}$
}

\begin{abstract}
- Additional supplemental material is published online only. To view, please visit the journal online (http://dx.doi.org/10. 1136/openhrt-2021-001702).

To cite: Sheehan MM, Saijo Y, Popovic ZB, et al.

Echocardiography in suspected coronavirus infection: indications, limitations and impact on clinical management. Open Heart 2021;8:e001702. doi:10.1136/

openhrt-2021-001702
\end{abstract}

Received 27 April 2021 Accepted 13 July 2021

A Check for updates

(C) Author(s) (or their employer(s)) 2021. Re-use permitted under CC BY-NC. No commercial re-use. See rights and permissions. Published by BMJ.

${ }^{1} \mathrm{EC}-10$, Cleveland Clinic Lerner College of Medicine, Case Western Reserve University, Cleveland, Ohio, USA

${ }^{2}$ Cardiovascular Medicine, Tokushima University Hospital, Tokushima, Japan

${ }^{3}$ Cardiovascular Medicine, Cleveland Clinic Main Campus Hospital, Cleveland, Ohio, USA

Correspondence to Dr Michael D Faulx; faulxm@ ccf.org

\section{ABSTRACT}

Objectives To describe the use of echocardiography in patients hospitalised with suspected coronavirus infection and to assess its impact on clinical management. Methods We studied 79 adults from a prospective registry of inpatients with suspected coronavirus infection at a single academic centre. Echocardiographic indications included abnormal biomarkers, shock, cardiac symptoms, arrhythmia, worsening hypoxaemia or clinical deterioration. Study type (limited or complete) was assessed for each patient. The primary outcome measure was echocardiography-related change in clinical management, defined as intensive care transfer, medication changes, altered ventilation parameters or subsequent cardiac procedures within 24 hours of echocardiography. Coronavirus-positive versus coronavirus-negative patient groups were compared. The relationship between echocardiographic findings and coronavirus mortality was assessed.

Results 56 patients were coronavirus-positive and 23 patients were coronavirus-negative with symptoms attributed to other diagnoses. Coronavirus-positive patients more often received limited echocardiograms ( $70 \%$ vs $26 \%, p=0.001)$. The echocardiographic indication for coronavirus-infected patients was frequently worsening hypoxaemia (43\% vs $4 \%$ ) versus chest pain, syncope or clinical heart failure ( $23 \%$ vs $44 \%)$. Echocardiography changed management less frequently in coronavirus-positive patients ( $18 \%$ vs $48 \%, p=0.01$ ). Among coronavirus-positive patients, 14 of 56 (25.0\%) died during hospitalisation. Those who died more often had echocardiography to evaluate clinical deterioration ( $71 \%$ vs $24 \%$ ) and had elevated right ventricular systolic pressures ( $37 \mathrm{~mm} \mathrm{Hg}$ vs $25 \mathrm{~mm} \mathrm{Hg}$ ), but other parameters were similar to survivors.

Conclusions Echocardiograms performed on hospitalised patients with coronavirus infection were often technically limited, and their findings altered patient management in a minority of patients.

\section{INTRODUCTION}

The COVID-19 pandemic has caused over 569000 deaths in the USA as of 26 April 2021. ${ }^{1}$ Pulmonary consequences of this disease, including acute respiratory distress

\section{Key questions}

What is already known about this subject?

- While it is known that echocardiographic markers of right ventricular (RV) impairment (RV enlargement and systolic dysfunction, increased estimated RV systolic pressure and abnormal right atrial and ventricular strain imaging) are predictive of increased mortality in patients hospitalised with COVID-19related pulmonary disease, indications for echocardiography in patients with COVID-19 or how the findings of these echocardiograms, which are often limited, point-of-care studies, impact patient management in real time compared with those without COVID-19 is less well characterised.

What does this study add?

- This study adds evidence that echocardiograms performed in COVID-19-infected patients are often of limited technical quality, and their results alter patient care in a minority of cases, particularly when the indication for the study lacks cardiac-specific findings or complaints.

How might this impact on clinical practice?

- These findings might impact clinical practice by suggesting that echocardiography in hospitalised patients with COVID-19 infection be used more judiciously, with the use of predefined indications and employing more complete studies performed by trained technicians wearing appropriate personal protective equipment. Such an approach might conserve resources and protect echocardiographic providers by limiting the number of 'low-yield' studies.

syndrome (ARDS), are significant contributors to morbidity and mortality. ${ }^{23}$ Observational studies suggest that COVID-19 may also affect the heart, leading to myocarditis, arrhythmias and acute coronary syndrome. ${ }^{4-6}$ The role of diagnostic cardiac testing in the management of these patients is still unclear.

Echocardiography is readily accessible and may be used to assess for functional cardiac injury and to monitor complications of both disease and treatment in patients with 
COVID-19. A study of 120 patients with COVID-19 by Li et al found that right ventricular (RV) longitudinal strain was a strong predictor of acute heart injury, ARDS and mortality. A large prospective international survey of 1216 COVID-19-positive patients from Dweck et al found that $55 \%$ had an abnormal echocardiogram. ${ }^{8}$ Echocardiographic findings changed management in only $33 \%$ of patients, most frequently in patients with pre-existing cardiac disease or elevated biomarkers. The American Society of Echocardiography recommends that echocardiography only be performed for patients infected with COVID-19 if expected to provide clinical benefit. ${ }^{9}$ Deciding which patients may benefit from echocardiography remains a challenge.

In this study, we characterise echocardiographic indications and parameters in a series of patients tested for COVID-19. We compare findings and management changes in patients with the disease to those who tested negative, with the goal to identify patients with COVID-19 who are more likely to benefit from echocardiography.

\section{METHODS}

\section{Study population}

This sample was taken from a prospective registry of all patients tested for COVID-19 at a large tertiary care centre. We included adult patients who were tested for COVID-19 from 11 March 2020 to 20 May 2020 on admission to the hospital and received an echocardiogram during their index COVID-19 hospitalisation. The patients were further grouped based on testing results. The patients were considered COVID-19-positive if confirmed by nasopharyngeal or oropharyngeal swabs, run with the use of the Centers for Disease Control and Prevention assay using Roche MagNA Pure extraction (Roche Life Science) and ABI 7500 DX PCR machines (Applied Biosystems/Thermo Fisher Scientific).

\section{Clinical data}

Demographics, comorbidities, presenting symptoms, medications, laboratory data within 24 hours of admission and outcomes were extracted from the electronic medical record. Laboratory testing measured on admission included blood urea nitrogen, creatinine, sodium, potassium, bicarbonate, glucose, white blood cell count, haemoglobin, haematocrit, platelet count, albumin, aspartate aminotransferase, alanine aminotransferase, C reactive protein, lactate, procalcitonin, interleukin-6, $\mathrm{N}$-terminal pro b-type natriuretic peptide, troponin $\mathrm{T}$, high-sensitivity troponin, creatine kinase, creatine kinase myocardial band, d-dimer, fibrinogen and ferritin. Outcomes of interest included need for intensive care, oxygen therapy, mechanical ventilation, shock, renal failure, use of vasopressors and mechanical circulatory support.

The index echocardiogram was identified for each patient as the first echocardiogram performed during hospitalisation. Echocardiogram type was classified as complete, limited technician study or point of care. Echocardiogram indication was also extracted from the medical record and divided into six categories: abnormal biomarkers, shock, cardiac symptoms, arrhythmia, worsening hypoxaemia or clinical deterioration. Patients could have multiple indications. Cardiac symptoms included syncope, physical signs of heart failure and cardiac arrest. An echocardiogram was considered to have changed management if transfer to intensive care, medication changes, alterations in ventilation parameters or subsequent cardiac procedures occurred within 24 hours in response to results and was determined by chart review.

\section{Echocardiographic assessment}

All patients underwent comprehensive baseline echocardiograms using commercial instruments (Philips Medical Systems, Bothell, Washington, USA; General Electric, Milwaukee, Wisconsin, USA). The extracted conventional echocardiographic parameters were measured, according to the current American Society of Echocardiography guidelines. ${ }^{1011}$ Echocardiographic analysis was completed using Syngo Dynamics (Siemens Healthcare, Erlangen, Germany). Left ventricular (LV) and left atrial (LA) volumes were measured using a modified biplane Simpson's method, and volume indexes were calculated as volume divided by body surface area. The peak early diastolic velocity and the peak early diastolic of mitral annular tissue motion velocities were measured. The ratio of mitral early inflow velocity to early mitral annular velocity $\left(\mathrm{E} / \mathrm{e}^{\prime}\right)$ ratio was calculated. Tricuspid lateral annular systolic velocity was assessed using tissue Doppler imaging from the apical four-chamber view. Tricuspid annular plane systolic excursion was measured as the systolic displacement of the tricuspid lateral annulus, recorded on M-mode imaging. Pulmonary artery systolic pressure was estimated from the peak velocity of tricuspid regurgitation jet using the modified Bernoulli equation plus right atrial (RA) pressure evaluated from the inferior vena cava size and collapsibility.

\section{Speckle tracking echocardiography (STE)}

Two-dimensional STE was used to characterise global longitudinal strain (GLS). Images for LV-GLS were acquired from apical $4 \mathrm{ch}$, 2ch and $3 \mathrm{ch}$ views, and images for LA-GLS, RV-GLS and RA-GLS were acquired from apical $4 \mathrm{ch}$ view. ${ }^{12}$ All images were analysed in a blinded manner, offline using vendor-independent STE software (Velocity Vector Imaging V.2; Siemens, Mountain View, California, USA). For each of the individual apical views, tracking was visually inspected throughout the systole to ensure adequate border tracking, and the endocardial contours were adjusted manually when necessary. The endocardial wall was divided into 6 segments in each view (18 segments for LV and 6 segments for LA, RV and RA), and the case was excluded if it was not feasible to track 2 or more segments in each view. In the setting of LV and $\mathrm{RV}$, negative peak strain value of the average of segmental 
strain curves was considered LV-GLS and RV-GLS, respectively. In the setting of LA and RA, zero strain reference was set at the $\mathrm{R}$ wave in the ECG, and positive peak strain value of the average of segmental strain curves was considered LA-GLS and RA-GLS, respectively. When tracing the borders of the LA endocardium, the confluences of the pulmonary veins and the LA appendage were excluded. All measurements were made by an experienced investigator blinded to clinical information.

\section{Statistical analysis}

Baseline data are presented as median (IQR), mean (SD) and number (percentage) where appropriate. Categorical variables were compared using the $\chi^{2}$ test or Fisher's exact test, and continuous variables were compared using Student's t-tests or Wilcoxon rank-sum test. Statistical testing was done using $\mathrm{R}$ V.4 (R Foundation for Statistical Computing, Vienna, Austria). A two-sided $p$ value of $<0.05$ was considered to be statistically significant.

\section{Patient and public involvement}

Our study involved data taken from a large prospective registry of patients hospitalised at our institution for suspected COVID-19 infection. Given the observational nature of our study, we did not directly involve patients or the public in its overall design.

\section{RESULTS}

A total of 79 patients were included in the study, and $56(71 \%)$ of these tested positive for COVID-19. Compared with those who tested negative, patients with COVID-19 were less likely to smoke (5.4\% vs $34.8 \%, \mathrm{p}=0.001$ ) but were otherwise similar in age, sex, ethnicity and comorbidities (table 1). Of those who tested negative for COVID-19, five (22\%) were diagnosed with bacterial pneumonia; five $(22 \%)$ were diagnosed with another infectious process; one (4\%) was diagnosed with viral pneumonia; four $(17 \%)$ were diagnosed with non-infectious pulmonary processes; seven $(30 \%)$ were diagnosed with heart failure exacerbation; and eight (35\%) were diagnosed with another cardiac process such as tamponade.

Patients with COVID-19 had lower troponin T (0.03 ng/ $\mathrm{mL}$ vs $0.13 \mathrm{ng} / \mathrm{mL}, \mathrm{p}=0.001$ ) and high-sensitivity troponin (33ng/L vs $72 \mathrm{ng} / \mathrm{L}, \mathrm{p}=0.02$ ) at admission compared with those who tested negative (table 2). They also had lower lactate levels $(1.6 \mathrm{mmol} / \mathrm{L}$ vs $2.8 \mathrm{mmol} / \mathrm{L}, \mathrm{p}=0.02)$ and white blood cell counts $\left(6.1 \times 10^{9} / \mathrm{L}\right.$ vs $10.6 \times 10^{9} / \mathrm{L}$, $\mathrm{p}=0.002)$. However, they were more likely to show pneumonia on a chest X-ray $(70 \%$ vs $22 \%, \mathrm{p}<0.001)$ and more often required oxygen therapy ( $89 \%$ vs $61 \%, \mathrm{p}=0.009$ ). Patients with COVID-19 had longer lengths of stay in both the hospital ( 17.7 days vs 6.1 days, $\mathrm{p}=0.002$ ) and the intensive care unit ( 15.9 days vs 5.0 days, $\mathrm{p}<0.001)$.

Patients with COVID-19 more often received limited technician echocardiograms ( $70 \%$ vs $26 \%, \mathrm{p}=0.001)$ later in their admission (median time from admission
Table 1 Demographic information and comorbidities stratified by COVID-19 status

\begin{tabular}{llll}
\hline & $\begin{array}{l}\text { COVID-19- } \\
\text { negative }\end{array}$ & $\begin{array}{l}\text { COVID-19- } \\
\text { positive }\end{array}$ & P value \\
\hline Demographics & 23 & 56 & \\
\hline $\mathrm{N}$ & $65.7 \pm 18.2$ & $62.5 \pm 15.2$ & 0.42 \\
\hline Age (mean \pm SD) & $17(73.9)$ & $37(66.1)$ & 0.68 \\
\hline $\begin{array}{l}\text { Gender (\% male) } \\
\text { Ethnicity (\%) }\end{array}$ & $9(39.1)$ & $25(44.6)$ & 0.65 \\
\hline $\begin{array}{l}\text { Black } \\
\text { Caucasian }\end{array}$ & $13(56.5)$ & $25(44.6)$ & \\
\hline Hispanic/Latino & $1(4.3)$ & $4(7.1)$ & \\
\hline $\begin{array}{l}\text { More than one } \\
\text { Alcohol use }\end{array}$ & $0(0)$ & $2(3.6)$ & \\
\hline $\begin{array}{l}\text { None } \\
\text { Moderate (<14 drinks/ }\end{array}$ & $9(39.1)$ & $18(32.1)$ & \\
week) & $10(43.5)$ & $34(60.7)$ & \\
\hline $\begin{array}{l}\text { Excessive (>14 drinks/ } \\
\text { week) }\end{array}$ & $4(17.4)$ & $4(7.1)$ & \\
\hline
\end{tabular}

Smoking

0.001

\begin{tabular}{|c|c|c|c|}
\hline Never & $5(21.7)$ & 29 (51.8) & \\
\hline Former & $10(43.5)$ & $24(42.9)$ & \\
\hline Active & $8(34.8)$ & $3(5.4)$ & \\
\hline Drug use (\%) & $3(13)$ & $8(14.3)$ & $>0.99$ \\
\hline \multicolumn{4}{|l|}{ Comorbidities (\%) } \\
\hline Cardiovascular disease & $14(60.9)$ & $25(44.6)$ & 0.29 \\
\hline CAD & $8(34.8)$ & $10(17.9)$ & 0.18 \\
\hline HFrEF & $6(26.1)$ & $9(16.1)$ & 0.47 \\
\hline $\mathrm{HFpEF}$ & $5(21.7)$ & $6(10.7)$ & 0.35 \\
\hline Aortic valve disease & $4(17.4)$ & $3(5.4)$ & 0.20 \\
\hline Mitral valve disease & $2(8.7)$ & $4(7.1)$ & $>0.99$ \\
\hline Atrial arrhythmias & $8(34.8)$ & $12(21.4)$ & 0.34 \\
\hline Ventricular arrhythmias & $2(8.7)$ & $2(3.6)$ & 0.71 \\
\hline PAD & $2(8.7)$ & $4(7.1)$ & $>0.99$ \\
\hline Aortopathy & $1(4.3)$ & $1(1.8)$ & $>0.99$ \\
\hline Pulmonary disease & $8(34.8)$ & $17(30.4)$ & 0.91 \\
\hline Stroke & $4(17.4)$ & $4(7.1)$ & 0.33 \\
\hline Hypertension & $14(60.9)$ & $37(66.1)$ & 0.86 \\
\hline Diabetes & $5(21.7)$ & $19(33.9)$ & 0.42 \\
\hline Cancer history & $5(21.7)$ & $9(16.1)$ & 0.78 \\
\hline CKD & $5(21.7)$ & $15(26.8)$ & 0.85 \\
\hline Gastrointestinal disease & $6(26.1)$ & $15(26.8)$ & $>0.99$ \\
\hline Neurological disease & $4(17.4)$ & $6(10.7)$ & 0.24 \\
\hline Psychiatric disease & $10(43.5)$ & $11(19.6)$ & 0.06 \\
\hline Sleep apnoea & $3(13)$ & $11(19.6)$ & 0.71 \\
\hline
\end{tabular}

CAD, coronary artery disease; CKD, chronic kidney disease; $\mathrm{HfpEF}$, heart failure with preserved ejection fraction; HfrEF, heart failure with reduced ejection fraction; PAD, peripheral arterial disease. 
Open Heart

Table 2 Laboratory testing and outcomes stratified by COVID-19 status

\begin{tabular}{|c|c|c|c|}
\hline & COVID-19-negative & COVID-19-positive & $P$ value \\
\hline \multicolumn{4}{|l|}{ Admission lab values, median (IQR) } \\
\hline BUN (mg/dL) & $25(20-44)$ & $24.5(15-45)$ & 0.65 \\
\hline Creatinine (mg/dL) & $1.37(0.92-2.02)$ & $1.42(0.91-1.94)$ & 0.74 \\
\hline $\operatorname{GFR}\left(\mathrm{mg} / \mathrm{min} / 1.73 \mathrm{~m}^{2}\right)$ & $31.5(23.5-48.0)$ & $46(30-60)$ & 0.09 \\
\hline Sodium (mmol/L) & $136(134.5-139.0)$ & $136.5(134.8-139.0)$ & 0.82 \\
\hline Potassium (mmol/L) & $4.2(3.85-4.65)$ & $4.2(3.9-4.5)$ & 0.66 \\
\hline Bicarbonate (mmol/L) & $21(19-24)$ & $23(20-25)$ & 0.46 \\
\hline Glucose (mg/dL) & $136(110.4-232.5)$ & $131(109.5-169.0)$ & 0.27 \\
\hline WBC $\left(\times 10^{9} / L\right)$ & $10.61(8.25-14.52)$ & $6.1(4.6-8.4)$ & 0.002 \\
\hline Haemoglobin $(\mathrm{g} / \mathrm{dL})$ & $12.5(10.95-14.25)$ & $12.9(10.9-14.2)$ & 0.67 \\
\hline Haematocrit (\%) & $37.8(33.6-43.35)$ & $39.3(35.0-42.5)$ & 0.78 \\
\hline Platelets (k/uL) & 206 (175.5-250) & $186.5(139.8-234.8)$ & 0.19 \\
\hline Albumin (g/dL) & $3.8(3.4-4.0)$ & $3.5(3.1-3.8)$ & 0.08 \\
\hline AST (U/L) & $30(24.5-64.0)$ & $39.5(31.9-74.5)$ & 0.22 \\
\hline ALT (U/L) & $20(17.5-37.0)$ & $26.5(19-41)$ & 0.60 \\
\hline CRP (mg/L) & $6.1(3.6-13.3)$ & $10.4(5.0-16.9)$ & 0.41 \\
\hline Lactate (mmol/L) & $2.8(1.6-3.95)$ & $1.6(1.2-2.3)$ & 0.02 \\
\hline Procalcitonin (ng/mL) & $1.39(0.75-2.92)$ & $0.34(0.18-0.66)$ & 0.04 \\
\hline IL-6 (pg/mL) & $115.6(67.8-19834.25)$ & $62(20.5-120.1)$ & 0.39 \\
\hline NT Pro-BNP (pg/mL) & $3712(305-9787)$ & 567 (181-2771) & 0.11 \\
\hline Troponin T (ng/L) & $0.13(0.04-0.4)$ & $0.03(0.01-0.05)$ & 0.001 \\
\hline Hs-troponin (ng/L) & $72(65.0-268.5)$ & $33(14-56)$ & 0.02 \\
\hline Creatine kinase (U/L) & $134.5(81.25-238)$ & 230 (98-461) & 0.31 \\
\hline CK-MB (ng/mL) & $3.3(2.3-26.65)$ & $3.8(2.2-4.5)$ & 0.56 \\
\hline D-dimer (ug/mL) & $2840(2150-8890)$ & $1250(610-3300)$ & 0.08 \\
\hline Fibrinogen (mg/dL) & 509 (343-679) & $541(434.5-667.5)$ & 0.64 \\
\hline Ferritin (ug/L) & 294.5 (167.62-506.35) & 704 (459-1470) & 0.13 \\
\hline \multicolumn{4}{|l|}{ Hospitalisation characteristics } \\
\hline Hospitalisation required (\%) & $23(100)$ & $56(100)$ & $>0.99$ \\
\hline Pneumonia on CXR (\%) & $5(21.7)$ & $39(69.6)$ & $<0.001$ \\
\hline Oxygen therapy & $14(60.9)$ & $50(89.3)$ & 0.009 \\
\hline ICU required & $22(95.7)$ & $43(76.8)$ & 0.10 \\
\hline ICU length of stay (mean $\pm S D$ ) & $5.0 \pm 4.1$ & $15.9 \pm 11.7$ & $<0.001$ \\
\hline NIPPV (\%) & $2(8.7)$ & $7(12.5)$ & 0.92 \\
\hline Mechanical ventilation (\%) & $9(39.1)$ & $33(58.9)$ & 0.18 \\
\hline Ventilation length (days) $(m e a n \pm S D)$ & $6 \pm 5.9$ & $13.9 \pm 11.8$ & 0.06 \\
\hline Prone ventilation (\%) & $0(0)$ & $12(21.4)$ & 0.04 \\
\hline Dialysis & $2(8.7)$ & $16(28.6)$ & 0.11 \\
\hline Shock (\%) & $13(56.5)$ & $31(55.4)$ & $>0.99$ \\
\hline Vasoactive drips (\%) & $16(69.6)$ & $33(58.9)$ & 0.53 \\
\hline Mechanical circulatory support & $3(13)$ & $4(7.1)$ & 0.69 \\
\hline Death (\%) & $8(34.8)$ & $14(25)$ & 0.55 \\
\hline
\end{tabular}

ALT, alanine aminotransferase; AST, aspartate aminotransferase; BUN, blood urea nitrogen; CK-MB, creatine kinase myocardial band; CRP, $C$ reactive protein; CXR, chest X-ray; GFR, glomerular filtration rate; ICU, intensive care unit; IL-6, interleukin-6; NIPPV, non-invasive positivepressure ventilation; NT Pro-BNP, N-terminal pro b-type natriuretic peptide; WBC, white blood cell. 


\begin{tabular}{|c|c|c|c|c|c|}
\hline & COVID-19-negative & & COVID-19-positive & & $P$ value \\
\hline $\mathrm{N}$ & $\begin{array}{l}\text { Available results } \\
\text { (out of 23) }\end{array}$ & & $\begin{array}{l}\text { Available results } \\
\text { (out of } 56 \text { ) }\end{array}$ & & \\
\hline Echocardiogram type & 23 & & 56 & & 0.001 \\
\hline Complete & & $14(60.9)$ & & $12(21.4)$ & \\
\hline Limited technician & & $6(26.1)$ & & $39(69.6)$ & \\
\hline Point of care & & $3(13)$ & & $5(8.9)$ & \\
\hline Echocardiogram indication & 23 & & 56 & & \\
\hline $\begin{array}{l}\text { Time from admission to } \\
\text { echocardiogram (days) }\end{array}$ & 23 & $1(1-1)$ & 56 & $2(1.0-4.3)$ & 0.003 \\
\hline \multicolumn{6}{|l|}{ Cardiac-specific } \\
\hline Abnormal biomarker & & $4(17.4)$ & & $9(16.1)$ & $>0.99$ \\
\hline Cardiogenic shock & & $3(13)$ & & $3(5.4)$ & 0.48 \\
\hline Cardiac signs & & $10(43.5)$ & & $13(23.2)$ & 0.13 \\
\hline Arrhythmia & & $2(8.7)$ & & $5(8.9)$ & $>0.99$ \\
\hline \multicolumn{6}{|l|}{ Not cardiac-specific } \\
\hline Worsening hypoxaemia & & $1(4.3)$ & & $24(42.9)$ & 0.002 \\
\hline Clinical deterioration & & $10(43.5)$ & & $20(35.7)$ & 0.70 \\
\hline Change in management & 23 & $11(47.8)$ & 56 & $10(17.9)$ & 0.01 \\
\hline LVEF \% (median, IQR) & 22 & $49(31.5-60.0)$ & 56 & $55(47.3-60.0)$ & 0.09 \\
\hline$\geq 50$ & & $11(47.8)$ & & 38 (67.9) & \\
\hline $40-49$ & & $4(17.4)$ & & $11(19.6)$ & \\
\hline $30-39$ & & $1(4.3)$ & & $3(5.4)$ & \\
\hline$<30$ & & $6(26.1)$ & & $4(7.1)$ & \\
\hline Diastolic function & 10 & & 43 & & 0.80 \\
\hline Grade 1 & & $6(26.1)$ & & $21(37.5)$ & \\
\hline Grade 2 & & $3(13)$ & & $7(12.5)$ & \\
\hline Grade $3 / 4$ & & $1(4.3)$ & & $2(3.6)$ & \\
\hline RV size & 19 & & 52 & & 0.18 \\
\hline Normal & & $17(73.9)$ & & $42(75)$ & \\
\hline Mildly dilated & & 0 & & $7(12.5)$ & \\
\hline Moderately dilated & & $2(8.7)$ & & $3(5.4)$ & \\
\hline Severely dilated & & $0(0)$ & & $1(1.8)$ & \\
\hline RV systolic function & 18 & & 51 & & 0.25 \\
\hline Normal & & $11(47.8)$ & & 39 (69.6) & \\
\hline Mildly reduced & & $3(13)$ & & $8(14.3)$ & \\
\hline Moderately reduced & & $3(13)$ & & $4(7.1)$ & \\
\hline Severely reduced & & $1(4.3)$ & & $1(1.8)$ & \\
\hline RVSP & 13 & $36(29-52)$ & 33 & $28(21-40)$ & 0.35 \\
\hline \multicolumn{6}{|l|}{ Strain \% } \\
\hline LV strain & 17 & $-11.8(-15.9$ to -6.3$)$ & 42 & $-14.5(-16.3$ to -11.6$)$ & 0.12 \\
\hline LA strain & 12 & $15.9(8.2-26.8)$ & 31 & $31.4(19.2-38.1)$ & 0.07 \\
\hline
\end{tabular}

Data displayed as median (IQR) or number (\%).

LA, left atrial; LV, left ventricular; LVEF, left ventricular ejection fraction; RV, right ventricular; RVSP, right ventricular systolic pressure.

to echocardiogram 2 days vs 1 day, $\mathrm{p}=0.003$ ) (table 3 ). The imaging indication for those who tested positive was frequently worsening hypoxaemia ( $43 \%$ vs $4 \%$ ), rather than cardiac symptoms including chest pain, syncope or clinical signs of heart failure $(23 \%$ vs $44 \%)$. Based on echocardiographic quality, we were able to analyse 58 


\section{A \\ COVID $19(+)$ Impactful Echocardiographic Studies ( $\mathrm{N}=10,18 \%$ of total)}

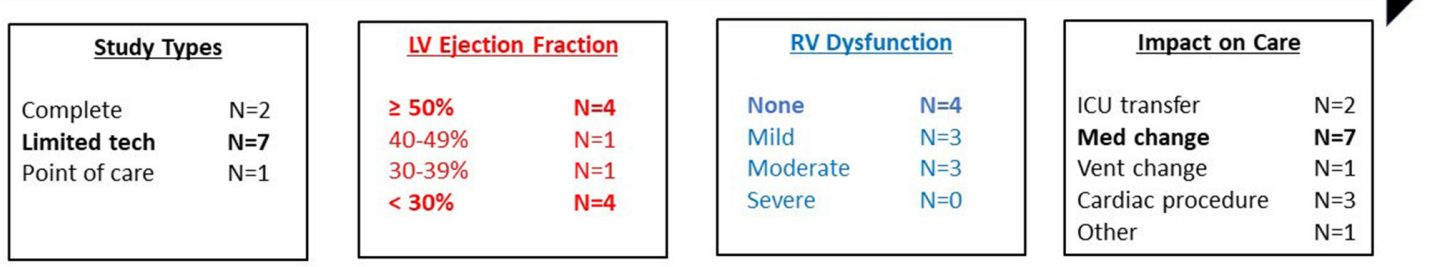

B

COVID 19 (-) Impactful Echocardiographic Studies ( $N=11,48 \%$ of total)

\begin{tabular}{|c|c|c|c|}
\hline \multicolumn{2}{|c|}{$\underline{\text { Study Types }}$} & \multicolumn{2}{|c|}{ LV Ejection Fraction } \\
\hline Complete & $\mathrm{N}=7$ & $\geq 50 \%$ & $\mathrm{~N}=4$ \\
\hline Limited tech & $\mathrm{N}=3$ & $40-49 \%$ & $\mathrm{~N}=1$ \\
\hline Point of care & $\mathrm{N}=1$ & $30-39 \%$ & $\mathrm{~N}=0$ \\
\hline & & $<30 \%$ & $N=7$ \\
\hline
\end{tabular}

\begin{tabular}{|ll|}
\hline \multicolumn{2}{|c|}{ RV Dysfunction } \\
None & $\mathrm{N}=4$ \\
Mild & $\mathrm{N}=1$ \\
Moderate & $\mathrm{N}=3$ \\
Severe & $\mathrm{N}=1$ \\
Not seen & $\mathrm{N}=2$ \\
\hline
\end{tabular}

\begin{tabular}{|ll|}
\hline \multicolumn{2}{|c|}{ Impact on Care } \\
ICU transfer & $\mathrm{N}=1$ \\
Med change & $\mathrm{N}=5$ \\
Vent change & $\mathrm{N}=0$ \\
Cardiac procedure & $\mathrm{N}=7$ \\
Other & $\mathrm{N}=0$ \\
\hline
\end{tabular}

Figure 1 Echocardiographic information, findings and resultant changes in care for COVID-19-positive (A) and COVID-19negative (B) patients. ICU, intensive care unit; LV, left ventricle; med, medication; RV, right ventricle; tech, technician; vent, ventilator.

patients (73\%) in LV-GLS, 18 patients (23\%) in RV-GLS, 43 patients $(54 \%)$ in LA-GLS and 25 patients $(32 \%)$ in RV-GLS. Echocardiographic parameters were closer to standard ranges for patients with COVID-19, including left ventricular ejection fraction (LVEF) (median of $55 \%$ vs $49 \%$, $\mathrm{p}=0.09)$, LV-GLS ( -14.5 vs $-11.8, \mathrm{p}=0.12)$ and LA strain (31.4 vs 15.9, $\mathrm{p}=0.07$ ) (table 3 ). There were $38(68 \%)$ patients with COVID-19 who had an ejection fraction $\geq 50 \%$, compared with 11 (48\%) of those without COVID19. Other parameters were difficult to measure on several patients due to image quality (online supplemental table 1). Echocardiographic results changed management less frequently in COVID-19-positive patients (18\% vs $48 \%$, $\mathrm{p}=0.01$; figure 1 ). Of the 10 COVID-19-positive patients who had alterations in management, 2 were transferred to intensive care; 7 had medication changes; 1 had a change in ventilation parameters; and 3 had a cardiac procedure performed.

\section{COVID-19-positive outcomes}

Of all COVID-19-positive patients, 14 out of 56 (25.0\%) died during the initial hospitalisation. Of those who died, all required intensive care, $11(79 \%)$ received mechanical ventilation, and $7(50 \%)$ required prone ventilation. Those who died were older (mean age 65.8 vs 61.4 years) and more often had pre-existing cardiovascular disease compared with those who survived ( $57 \%$ vs $41 \%)$. Echocardiographic parameters for those who died compared with those who tested positive but survived their initial hospitalisation are presented in table 4 . Those who died more often had echocardiography performed due to clinical deterioration $(71 \%$ vs $24 \%)$ and had elevated right ventricular systolic pressures (RVSPs) (37 mm $\mathrm{Hg}$ vs $25 \mathrm{~mm} \mathrm{Hg}$ ), but other parameters were similar between groups, including LVEF ( $57.5 \%$ vs $55.0 \%)$.

\section{DISCUSSION}

In this observational study of 79 patients tested for COVID-19 who had echocardiographic imaging performed at a large tertiary care centre, we found that the vast majority of echocardiograms in COVID-19positive patients were unremarkable. The majority of COVID-19-positive patients in our sample had LVEFs of $\geq 50 \%$ and normal RV systolic function. This is different from reports from Dweck et al, who found that $55 \%$ of echocardiograms among COVID-19-positive patients were abnormal. This is surprising, as our patient sample has a higher burden of underlying cardiovascular disease and other comorbidities. However, patients in our sample often had cardiac imaging performed for non-cardiacspecific signs, rather than signs of heart failure or elevated biomarkers. This may explain the lower frequency of echocardiographic abnormalities in our study.

Compared with patients who were admitted to the hospital and ultimately tested negative for COVID-19, patients with COVID-19 received limited technician studies more frequently than a complete echocardiogram. There are several possible reasons for this finding. Imaging conditions may have been different among groups, as a larger number of COVID-19-positive patients underwent dialysis and mechanical ventilation during hospitalisation. These may have posed an additional challenge for complete examination at bedside for COVID19-positive patients. It is also possible that clinicians used echocardiograms differently between the two groups and required less information from the COVID-19-positive group to make clinical decisions. During the study period, hospitals tried to minimise staff exposure to patients with COVID-19, which may also contribute to differences in imaging. Amid concerns of prolonged exposure for technicians and limited personal protective equipment, these 
Table 4 Echocardiographic parameters for COVID-19positive patients stratified by survival

\begin{tabular}{|c|c|c|c|}
\hline & Survivors & Deaths & $P$ value \\
\hline $\mathrm{N}$ & 42 & 14 & \\
\hline \multicolumn{3}{|c|}{ Echocardiogram type } & 0.59 \\
\hline Complete & $10(23.8)$ & $2(14.3)$ & \\
\hline $\begin{array}{l}\text { Limited } \\
\text { technician }\end{array}$ & $29(69)$ & $10(71.4)$ & \\
\hline Point of care & $3(7.1)$ & $2(14.3)$ & \\
\hline
\end{tabular}

Echocardiogram indication

\begin{tabular}{|c|c|c|c|}
\hline \multicolumn{4}{|l|}{ Cardiac-specific } \\
\hline $\begin{array}{l}\text { Abnormal } \\
\text { biomarker }\end{array}$ & $7(16.7)$ & $2(14.3)$ & $>0.99$ \\
\hline $\begin{array}{l}\text { Cardiogenic } \\
\text { shock }\end{array}$ & $2(4.8)$ & $1(7.1)$ & $>0.99$ \\
\hline Cardiac signs & $11(26.2)$ & $2(14.3)$ & 0.58 \\
\hline Arrhythmia & $4(9.5)$ & $1(7.1)$ & $>0.99$ \\
\hline \multicolumn{4}{|l|}{ Not cardiac-specific } \\
\hline $\begin{array}{l}\text { Worsening } \\
\text { hypoxaemia }\end{array}$ & 18 (42.9) & $6(42.9)$ & $>0.99$ \\
\hline $\begin{array}{l}\text { Clinical } \\
\text { deterioration }\end{array}$ & $10(23.8)$ & $10(71.4)$ & 0.004 \\
\hline $\operatorname{LVEF}(\%)$ & $55(48-60)$ & $57.5(46.0-63.8)$ & 0.80 \\
\hline Diastolic function & & & 0.57 \\
\hline Grade 1 & $17(40.5)$ & $4(28.6)$ & \\
\hline Grade 2 & $5(11.9)$ & $2(14.3)$ & \\
\hline Grade 3/4 & $1(2.4)$ & $1(7.1)$ & \\
\hline RV size & & & 0.69 \\
\hline Normal & $32(76.2)$ & $10(71.4)$ & \\
\hline Mildly dilated & $6(14.3)$ & $1(7.1)$ & \\
\hline $\begin{array}{l}\text { Moderately } \\
\text { dilated }\end{array}$ & $3(7.1)$ & $0(0)$ & \\
\hline $\begin{array}{l}\text { Severely } \\
\text { dilated }\end{array}$ & $1(2.4)$ & $0(0)$ & \\
\hline \multicolumn{2}{|l|}{ RV systolic function } & & 0.68 \\
\hline Normal & $30(71.4)$ & $9(64.3)$ & \\
\hline Mildly reduced & $6(14.3)$ & $2(14.3)$ & \\
\hline $\begin{array}{l}\text { Moderately } \\
\text { reduced }\end{array}$ & $4(9.5)$ & $0(0)$ & \\
\hline $\begin{array}{l}\text { Severely } \\
\text { reduced }\end{array}$ & $1(2.4)$ & $0(0)$ & \\
\hline RVSP & $25(20.3-38.9)$ & $37(30.5-65.0)$ & 0.04 \\
\hline \multicolumn{4}{|l|}{ Strain \% } \\
\hline LV strain & $-14.4(-16.5$ to -11.5$)$ & $-14.9(-15.6$ to -13.4$)$ & 0.46 \\
\hline LA strain & $30.5(19.9-37.8)$ & $38.5(23.2-45.0)$ & 0.29 \\
\hline
\end{tabular}

Data displayed as median (IQR) or number (\%).

LA, left atrial; LV, left ventricular; LVEF, left ventricular ejection fraction; $\mathrm{RV}$, right ventricular; RVSP, right ventricular systolic pressure.

findings align with efforts to minimise risk for healthcare workers. However, as many of the echocardiographic parameters were within normal ranges for the COVID19-positive cohort of patients, these studies may not be critical to management or clinical decision-making, particularly among those without cardiac-specific signs of injury. Echocardiographic results changed management for relatively few patients with COVID-19 in this study, which is consistent with what others have found. ${ }^{13}$ Echocardiography should only be performed if there is an expected benefit in the management of the patient, and risk to healthcare workers and the patient should be carefully considered. In patients with suspected myocardial injury, further investigation may be warranted, as this is important for prognosis and may alter management for a select group of patients. ${ }^{14}$

We also found that patients who tested negative for COVID-19 and received echocardiograms were more ill than those who tested positive and received similar imaging. This is consistent with results from a similar study from Guo et al, in which clinical and echocardiographic parameters in a group of patients with COVID-19 were compared with retrospective analysis of echocardiograms from flu-positive patients. They found that flu-positive patients had a higher prevalence of LV failure and lower cardiac indices than patients with COVID-19. ${ }^{14}$ Similarly, patients in our study without COVID-19 often had greater lab abnormalities on initial presentation, such as elevated white blood cell counts and lactate levels, often required intensive care and had an in-hospital mortality rate of $35 \%$. Their echocardiographic parameters were often worse. We studied patients with and without COVID-19 during the same time period, allowing us to compare changes in clinical practice and decision-making directly. Our findings suggest that the threshold for ordering an echocardiogram based on clinical presentation was lower for COVID-19-positive patients.

Finally, we found that elevated RVSP was frequently observed in patients with COVID-19 who died. RV failure is a known complication of severe ARDS, and resulting haemodynamic instability is an important cause of mortality in these patients. ${ }^{15}$ Our findings are consistent with previous reports in the COVID-19 population. ${ }^{1617}$

There are limitations to our study. There are likely differences between patients with COVID-19 for whom an echocardiogram was ordered and those who did not have one during their hospitalisation, which limits the generalisability of our study. There may be factors that we did not measure in our study that led to additional evaluation and imaging. There may also be undocumented changes in management as a result of the echocardiographic results which were not captured in our analysis. It is possible that results helped clinicians decide not to escalate care further. The quality of the images that were used for analysis also varied, which impacted the sample size for certain echocardiographic parameters. The low quality of images in the COVID-19-positive patients may have made it difficult for clinicians to make management decisions based on these results, which would lead us to underestimate changes in management. However, as the majority of images provided basic information regarding ventricular function, we believe that the reported normal 
findings in these images are the reason for a lack of clinical action.

Our study is the first to our knowledge to compare echocardiographic indications and parameters in a cohort of patients tested for COVID-19 during the same time period, allowing us to compare directly for changes in clinical practice and diagnostics.

In summary, we found that the majority of echocardiograms performed in a cohort of COVID-19-positive patients during hospitalisation were unremarkable. These studies did not alter management in most cases, particularly among those without cardiac-specific signs of injury. Patients with COVID-19 who died were more likely to show RV dysfunction on echocardiography, consistent with the pathophysiology of SARS-CoV-2. Our findings suggest that hospital systems might consider limiting the use of echocardiography in patients with COVID-19 to those with unambiguous evidence of cardiovascular complication or injury where study findings are most likely to result in meaningful management changes that would better justify the risk of exposing echocardiographic technicians and physician sonographers to potential infection. For COVID-19-positive patients undergoing echocardiography, a point of emphasis should be made to obtain accurate estimates of RVSP and RV systolic function, given the negative prognostic implications of pulmonary hypertension and RV dysfunction in patients with COVID-19 infection.

\section{Twitter Michael D Faulx @MichaelFaulx}

Contributors MMS was responsible for study data acquisition, offline echocardiographic measurements, primary statistical analyses and manuscript draft preparation. YS was responsible for offline echocardiographic measurements, Methods section preparation and final manuscript editing. ZBP was responsible for study design input, supervision of offline echocardiographic measurement and final manuscript editing. MDF was responsible for primary study design, institutional review board submission, study database creation, study data acquisition, abstract authorship and final manuscript editing, and was the overall content guarantor.

Funding The authors have not declared a specific grant for this research from any funding agency in the public, commercial or not-for-profit sectors.

Competing interests None declared.

Patient consent for publication Not required.

Ethics approval The study protocol was reviewed and approved by the Cleveland Clinic institutional review board (IRB 20-480) and deemed both ethical and low risk to study participants. Due to its low-risk nature, the study received consent exempt status. Our registry was subject to institutional review and was granted an exemption for informed consent owing to the use of deidentified patient data and minimal patient risk.

Provenance and peer review Not commissioned; externally peer reviewed.

Data availability statement Deidentified patient data are available in a passwordprotected RedCap database and as deidentified studies within a clinical imaging server, accessible by the authors. These data can be made available upon reasonable request.
Open access This is an open access article distributed in accordance with the Creative Commons Attribution Non Commercial (CC BY-NC 4.0) license, which permits others to distribute, remix, adapt, build upon this work non-commercially, and license their derivative works on different terms, provided the original work is properly cited, appropriate credit is given, any changes made indicated, and the use is non-commercial. See: http://creativecommons.org/licenses/by-nc/4.0/.

ORCID iDs

Yoshihito Saijo http://orcid.org/0000-0002-1621-0200

Michael D Faulx http://orcid.org/0000-0002-6946-778X

\section{REFERENCES}

1 CDC. Coronavirus disease 2019 (COVID-19) in the U.S. centers for disease control and prevention, 2019. Available: https://www.cdc. gov/covid-data-tracker [Accessed 05 Nov 2020].

2 Huang C, Wang Y, Li X, et al. Clinical features of patients infected with 2019 novel coronavirus in Wuhan, China. Lancet 2020;395:497-506.

3 Wu C, Chen X, Cai Y, et al. Risk factors associated with acute respiratory distress syndrome and death in patients with coronavirus disease 2019 pneumonia in Wuhan, China. JAMA Intern Med 2020;180:934-11.

4 Shi S, Qin M, Shen B, et al. Association of cardiac injury with mortality in hospitalized patients with COVID-19 in Wuhan, China. JAMA Cardiol 2020;5:802-10.

5 Zhou F, Yu T, Du R, et al. Clinical course and risk factors for mortality of adult inpatients with COVID-19 in Wuhan, China: a retrospective cohort study. Lancet 2020;395:1054-62.

6 Bhatla A, Mayer MM, Adusumalli S, et al. COVID-19 and cardiac arrhythmias. Heart Rhythm 2020;17:1439-44.

7 Li Y, Li H, Zhu S, Shuangshuang Z, et al. Prognostic value of right ventricular longitudinal strain in patients with COVID-19. JACC Cardiovasc Imaging 2020;13:2287-99.

8 Dweck MR, Bularga A, Hahn RT, et al. Global evaluation of echocardiography in patients with COVID-19. Eur Heart J Cardiovasc Imaging 2020;21:949-58.

9 Kirkpatrick JN, Mitchell C, Taub C, et al. Ase statement on protection of patients and echocardiography service providers during the 2019 novel coronavirus outbreak: endorsed by the American College of cardiology. J Am Coll Cardiol 2020;75:3078-84.

10 Lang RM, Badano LP, Mor-Avi V, et al. Recommendations for cardiac chamber quantification by echocardiography in adults: an update from the American Society of echocardiography and the European association of cardiovascular imaging. J Am Soc Echocardiogr 2015;28:1-39.

11 Mitchell C, Rahko PS, Blauwet LA, et al. Guidelines for performing a comprehensive transthoracic echocardiographic examination in adults: recommendations from the American Society of echocardiography. J Am Soc Echocardiogr 2019;32:1-64.

12 Badano LP, Kolias TJ, Muraru D, et al. Standardization of left atrial, right ventricular, and right atrial deformation imaging using two-dimensional speckle tracking echocardiography: a consensus document of the EACVI/ASE/Industry Task force to standardize deformation imaging. Eur Heart $J$ Cardiovasc Imaging 2018;19:591-600.

13 Benyounes N, Van Der Vynckt C, Tibi S, et al. Echocardiography in confirmed and highly suspected symptomatic COVID-19 patients and its impact on treatment change. Cardiol Res Pract 2020;2020:1-9. doi:10.1155/2020/4348598

14 Guo T, Fan Y, Chen M, et al. Cardiovascular implications of fatal outcomes of patients with coronavirus disease 2019 (COVID-19). JAMA Cardiol 2020;5:811-8.

15 Evrard B, Goudelin M, Montmagnon N, et al. Cardiovascular phenotypes in ventilated patients with COVID-19 acute respiratory distress syndrome. Crit Care 2020;24:236.

16 Zochios V, Parhar K, Tunnicliffe W, et al. The right ventricle in ARDS. Chest 2017;152:181-93.

17 Argulian E, Sud K, Vogel B, et al. Right ventricular dilation in hospitalized patients with COVID-19 infection. JACC Cardiovasc Imaging 2020;13:2459-61. 